Journal of Universal Language 14-1

March 2013, 55-84

\title{
An Interpretation Domain with Context-Dependent Events
}

\author{
Eun-Joo Kwak \\ Sejong University
}

\begin{abstract}
I address the interpretations of verbal predicates, focusing on the structural similarities between the domains of objects and events. Based on Davidson's (1967) theorum that events are individuals just like objects, I start from a proper interpretation domain for nominal interpretations and expand it to verbal interpretations. I critically review Rothstein (2008), which takes an interpretation domains suggested by Chierchia (1998). To resolve problems in the earlier approaches, I resort to a vague interpretation domain of Chierchia (2010) and suggest an event structure for proper interpretations of verbal predicates in accord with Vendler's (1967) classification.
\end{abstract}

Keywords: predicate, aspectual class, state, activity, achievement, accomplishment, event, interpretation domain, atom, nonatom, vagueness

\footnotetext{
Eun-Joo Kwak

Department of English Language and Literature, Sejong University

98 Gunja-dong, Gwangjin-gu, Seoul 143-747, Korea

Phone: +82-2-3408-3633; Email: ejkwak@sejong.ac.kr
}

Received November 12, 2012; Revised January 5, 2013; Accepted February 10, 2013. 


\section{Introduction}

Sentence interpretations are possible when an interpretation domain is properly modeled. In earlier theories of semantics, an interpretation domain is assumed to consist of individuals for nominal predicates. This simple domain is later extended with 'events' for the interpretations of verbal predicates. Following Davidson (1967), events are assumed to be individuals just like objects for nominal interpretations. The assumption of events as individuals leads to the argument that the event structure is more or less the same as that of objects.

When events are introduced in semantics, nominal predicates are mainly construed with discrete objects. However, when semantic interests are broadened to the interpretations of mass and plural expressions, an interpretation domain needs to be expanded to deal with them. Hence, not only atoms for count interpretations but also nonatoms for mass interpretations are postulated in a domain, and a lattice structure is adopted to provide a uniform structure for both atoms and nonatoms.

In the revised domain of atoms and nonatoms, events for verbal interpretations should also be reconsidered for the paralleled structures of objects and events. For this purpose, Rothstein (2008) adopts Chierchia's (1998) argument for the lexical plurality of mass terms and puts forward an analysis for different event interpretations via verbal categories.

In this study, I will point out theoretical problems with Rothstein (2008) and suggest an alternative analysis for verbal interpretations. I will resort to an interpretation domain with context-dependent atoms for mass interpretations as proposed by Chierchia (2010) and propose that state and activity predicates are construed to denote sets of context-dependent events. 


\section{An Interpretation Domain with Individuals and Events}

\subsection{The Introduction of Event in an Interpretation Domain}

Before the insightful argument of Davidson (1967), verbal predicates are assumed to denote sets of individuals or relations between individuals. For instance, the meaning of run is to denote a set of runners, namely $\lambda x\left[\operatorname{run}^{\prime}(\mathrm{x})\right]$. Davidson argues that verbal interpretations based on nominal arguments are not suitable to account for diverse linguistic phenomena such as verbal modification, perceptual constructions, and adverbial quantification. Thus, he suggests that a verbal predicate has an extra argument of 'event' to represent its action.

(1) a. $[[\operatorname{run}]]=\lambda x \lambda e\left[\operatorname{run}^{\prime}(x)(e)\right]$

b. $[[$ John runs $]]=\exists \mathrm{e}\left[\operatorname{run}^{\prime}(\mathrm{j})(\mathrm{e})\right]$

As exemplified in (1a), run is construed to be a two-place predicate taking an object and an event in the Davidsonian theory (cf. Parsons 1990, Lasersohn 1995, Schein 1995, Landman 1996 among others). An existential closure is assumed on the sentential level, and thus a sentence is interpreted to assert the existence of an event the type of which is described by the sentence. As in (1b), the sentence John runs describes an event type of John's running and asserts the existence of an event belonging to this event type when the existential closure applies. Now, an interpretation domain is divided into two categories of entities, i.e., objects and events.

Davidson further argues that events are individuals just like objects for nominal interpretations. Since the event of John's running is an individual, it may be nailed down to a specific event by the existential closure, and its properties are more specified by 
modification. The individual properties of events also make it possible to quantify them by sentential operators. Now that events are assumed to be individuals, their structure should be similar to that of objects. This means that any change in the object domain triggers a change in the event domain.

\subsection{Vendler's Classification of Predicates}

Based on semantic properties such as duration over time, change, set terminal point, and homogeneity, Vendler (1967) classifies verbs into four categories: states, activities, achievements, and accomplishments. Here are some examples for the categories. ${ }^{1}$

(2) a. state: love, know, believe

b. activity: run, walk, push a cart, write letters

c. achievement: arrive, die, notice, realize

d. accomplishment: build a house, run a mile, walk to the car, grow up

One of the semantic properties in defining the categories is the progressive aspect. States and achievements do not naturally occur in the progressive while activities and accomplishments do.

(3) a. \#John is believing in the devil.

b. \#John is noticing the picture.

1 According to Vendler (1967), activities and accomplishments are processes going on in time. An essential feature of accomplishments is that they incorporate a terminal point to complete their event type. Although running does not have a set terminal point, running a mile has a terminal point, i.e., a mile. States and activities are homogeneous while accomplishments are not. Any part of running is running. However, the initial part of building a house cannot be the same as final stage of building it. Finally, while states do not entail any change in their states, the ontological properties of activities include indefinite change and those of achievements and accomplishments include definite change. 
c. John is running.

d. John is building a house.

The awkwardness of (3a) and (3b) sharply contrasts with the naturalness of (3c) and (3d). Another major distinction is that achievements and accomplishments occur with telic modifiers such as in half an hour whereas atelic modifiers like for half an hour suit for states and activities.

(4) a. John believed in the devil \#in half an hour/for several years.

b. John run \#in half an hour/for half an hour.

c. John noticed the picture in half an hour/\#for half an hour.

d. John built a house in two weeks/\#for two weeks.

This difference leads to the distinction of telic and atelic predicates. Achievements and accomplishments are grouped as telic predicates whereas states and activities are labled as atelic predicates. The four categories are also distinguished in stativity. Only states are assigned the stative feature, and the other three categories are classified as dynamic predicates.

Vendler's classification of verbal predicates has been widely used in the analyses of grammatical phenomena. Diverse patterns of grammatical aspects are properly described via verbal categories. The syntax-semantics interface is also explained well, especially related with argument structures and thematic roles. Both language-specific and universal descriptions are possible with the notion of verbal categories. Depending on languages, the choice of auxiliary verbs is also affected by verbal categories.

Davidson focuses on the dynamic properties of events, and thus his original argument for events does not postulate event arguments for states. However, his proposal for events is expanded to cover the semantics of states by his followers because states 
also show similar interpretive patterns in modification, perceptual structures, and adverbial quantification. Hence, we assume that predicates are assigned event arguments regardless of their lexical categories.

\subsection{An Interpretation Domain with Atoms and Nonatoms}

When semantic interests are broadened to cover the semantics of mass and plural NPs (noun phrases), a revision of the interpretation domain is inevitable. To meet this necessity, Link (1983) adopts a lattice-theoretic structure to model distinctions between count and mass nouns properly. A join semi-lattice structure is considered as appropriate to implement the cumulativity and homogeneity of mass nouns. Link divides an interpretation domain into two subdomains, one with atoms and the other with nonatoms. Singular count nouns are assumed to denote sets of atoms while the denotations of mass nouns are generated from nonatoms to reflect their homogeneity. Link further argues for a similarity between mass and plural NPs, i.e., cumulativity, and implements it by the join operation ' + ' in the lattice structure. Any two atoms may be joined to make a plural entity of sum. For example, two atomic individuals $\mathrm{j}$ for John and $\mathrm{m}$ for Mary are joined to make a sum $\mathrm{j}+\mathrm{m}$ to denote the plural John and Mary. Furthermore, any two sums are joined to make a larger sum, which shows the cumulativity of plurals. Similarly, any two materials of mass nouns are joined together to make a larger sum of material. Finally, Link introduces the part-of relation ' $\leq$ ' to reflect homogeneity. A sum $\mathrm{j}+\mathrm{m}$ has two parts $\mathrm{j}$ and $\mathrm{m}$, the relation of which is defined by $\leq: \mathrm{j} \leq \mathrm{j}+\mathrm{m}$ and $\mathrm{m} \leq \mathrm{j}+\mathrm{m}$. Likewise, a material sum of water $\mathrm{w}_{1}+\mathrm{w}_{2}$ are divided into two parts $\mathrm{w}_{1}$ and $\mathrm{w}_{2}$ by the part-of relation.

Bach (1986) extends Link's (1983) lattice-theoretic domain of individuals to the event domain by pointing out structural analogies between nominal and verbal predicates. ${ }^{2}$ States and 
activities are divisive and cumulative. The state of knowing may be divided into smaller parts of knowing states, and the event of running may also be divided into a number of subevents, i.e., smaller parts of running events. Similarly, smaller parts of knowing or running may be joined to make a larger sum of state or event. However, the events of achievement or accomplishments are not divisive and cumulative. The event of achievements like arrive is not divisive due to their instantaneous property, and parts of building a house do not have the same event type of building a house. Likewise, two events of arriving do not denote a cumulative event of arriving, and two events of building a house are not mapped to an atomic event of building a house.

The analysis of an individual structure is based on the spatial part-of relation of individuals wheres that of an event structure hinges on the temporal part-of relation of events. Given the difference between individuals and events, the close comparisons between the denotations of nominal and verbal predicates are exemplified as follows:

(5) a. MASS \& PLURAL NPs: wine, apples

b. SINGULAR COUNT NPs: an/the/one apple

c. QUANTIFIED \& MEASURE NPs: five/all (the) apples, a glass of wine

(6) a. STATE: Mary was in New York.

b. ACTIVITY: Mary drank wine.

c. ACHIEVEMENT: Mary arrived.

d. ACCOMPLISHMENT: Mary drank a glass of wine.

Nominal predicates are divided into three categories as in (5). Mass and plural NPs denote divisive and cumulative entities.

2 Although Bach (1986) uses the term 'process' instead of activity, we will use activity in summarizing Bach's analysis to avoid unnecessary confusion. 
Singular count NPs have atomic denotations which do not have subparts while quantified and measure NPs denote atoms which have parts internally. Atelic predicates of states and activities share ontological properties with mass and plural NPs such as divisivity and cumulativity. Achievements are instantaneous predicates, denoting sets of atomic events. Hence, they are more like singular count NPs in their denotations. Accomplishments involve temporal duration to complete their event types, and thus they are closely compared with quantified or measure NPs.

Krifka (1986, 1992) also argues for the mapping relation between nominal and verbal predicates, making use of thematic relations between nominal arguments and events. The telicity of accomplishments is determined by the quantity of their incremental themes. For instance, an eating event is categorized as an accomplishment when it is accompanied by a quantized argument like an apple or three apples. Moreover, the eating event undergoes successive changes as its theme, namely an apple or three apples, is used up gradually. In other words, homomorphism holds between the extent of the spatial portions of the theme and the extent of the running time of the event.

\section{Rothstein's (2008a) Analysis of Events}

\subsection{Ontological Problems with the Interpretation Domain}

Divisibility and cumulativity are useful notions to distinguish the nonatomic denotations of mass nouns from atomic denotations of count nouns. However, when the infinite nature of divisibility is not properly curbed, it ends up with awkward results. Although a portion of water may be divided into smaller portions of water without losing its property, not any parts of water may be considered as water. For example, a few molecules of water do 
not count as water in most cases, and two hydrogen atoms and one oxygen atom, which are divided from one molecule of water, are not considered as having water properties. This is a 'minimality' problem of nonatoms, i.e., defining minimal entities for the nonatoms of substances.

The minimality of nonatoms turns out to be a more serious problem because it is affected by contexts. A bowl of rice is rice, and a half bowl or a spoon of rice usually counts as rice. However, judgments may vary for a single grain of rice. In ordinary situations, e.g., judging whether a kid finished his meal, a grain of rice is not considered as large enough to make a substance of rice. In contrast, a grain of rice may be considered as rice in an experimental situation. Rice flour, which is smaller than a grain of rice, may also be understood as rice in analyzing the material composition of food.

Another problem related with an interpretation domain is that 'semantic atomicity' is a separate notion from 'natural atomicity.' Chierchia (1998) spares extensive space to argue for the arbitrariness of semantic atomicity. Natural atomicity is determined by the physical structure of entities. If some object is spatially separated from other objects in nature and its identity is constantly preserved, it is naturally atomic. The denotation of apple is naturally atomic because apples have constant identities that are spatially separated from other objects. However, the material of water is not naturally atomic because there is no specific portion of water that is always separated from other entities in nature. At first glance, natural atomicity appears to determine the atomicity of nominal denotations. However, Chierchia argues that the semantic atomicity of nominal denotations may be arbitrary. In spite of having similar perceptual salience, rice is mass while lentil is count. A pair of count and mass nouns (e.g., shoe/footwear and change/coin) may be lexicalized for the same objects.

Finally, the lexical categories of count and mass nouns may be 
shifted.

(7) a. There was dog splattered all over the road.

b. They ordered three beers at the cafe.

The count noun $d o g$ is used as mass in (7a) whereas the mass noun beer is used as count in (7b). Dog in (7a) denotes the flesh material of dog rather than an individual of dog. Three beers in (7b) denotes three servings (e.g., bottles or glasses) of beer rather than the substance of beer. These are examples of meaning shifts by contexts. To deal with these meaning shifts, two homomorphism functions are incorporated in a domain: the 'universal grinding' and the 'universal packaging.' The universal grinding function maps atoms to their material counterparts while the universal packaging function maps material parts to atoms that are made of the materials. Note that this type of meaning shifts are also affected by contexts.

(8) a. There is apple in the soup.

b. ??There is table all over the floor.

(9) a. I drank three beers.

b. ??I drank three waters.

c. \#I drank three bloods.

In spite of the same countability for apple and table, the meaning shift of table is less natural than that of apple. Similarly, different degrees of acceptability are observed with the shifted readings of mass nouns as in (9).

The problems discussed in this section lead to the argument that an interpretation domain is not geared to model the physical world as it is. It should be properly structured to deal with diverse semantic issues. 


\subsection{A Revised Domain of Individuals: Rothstein (2010)}

Arguing against Link's (1983) separate domains of atoms and nonatoms, Chierchia (1998) proposes a single domain with atoms for both count and mass noun interpretations. Based on the distinct notions of natural and semantic atomicity as surveyed in the previous section, Chierchia argues that count and mass nouns are distinctively construed via their morpho-syntactic properties such as pluralization and direct modification by cardinal numerals. The natural atomicity of objects is not a crucial factor in determining the atomicity of nominal denotations. ${ }^{3}$

According to Chierchia, mass nouns denote plural entities. Suppose that there are three pieces of furniture in a given context: two chairs $\mathrm{c}_{1}$ and $\mathrm{c}_{2}$, and a table $\mathrm{t}_{1}$. A singular term piece of furniture denotes a set of these three pieces as in (10a).
a. $[[$ piece of furniture $]]=\left\{\mathrm{c}_{1}, \mathrm{c}_{2}, \mathrm{t}_{1}\right\}$
b. $[$ pieces of furniture $]]=\left\{\mathrm{c}_{1}, \mathrm{c}_{2}, \mathrm{t}_{1}, \mathrm{c}_{1}+\mathrm{c}_{2}, \mathrm{c}_{1}+\mathrm{t}_{1}, \mathrm{c}_{2}+\mathrm{t}_{1}\right.$, $\left.\mathrm{c}_{1}+\mathrm{c}_{2}+\mathrm{t}_{1}\right\}$
c. $[[$ furniture $]]=\left\{\mathrm{c}_{1}, \mathrm{c}_{2}, \mathrm{t}_{1}, \mathrm{c}_{1}+\mathrm{c}_{2}, \mathrm{c}_{1}+\mathrm{t}_{1}, \mathrm{c}_{2}+\mathrm{t}_{1}, \mathrm{c}_{1}+\mathrm{c}_{2}+\mathrm{t}_{1}\right\}$

Its plural counterpart pieces of furniture denotes a set closed under the sum operation in (10b). Since the mass collection furniture takes atomic members, its denotation in (10c) is identical to the plural pieces of furniture. Based on the equal denotations of plurals and mass collections, Chierchia argues that mass nouns are singular morpho-syntactically but plural in their lexicon. Since

3 Two categories of nouns are classified as mass nouns according to their morpho-syntactic properties, i.e., substances with no minimal parts (e.g., water and $m u d$ ) and mass collections that may have atomic members (e.g., furniture and silverware). Most of earlier approaches focus on the non-atomic nature of substances and argue for an atomless domain for mass nouns. In contrast, Chierchia (1998) starts from the atomicity of mass collections and proposes plural denotations for mass nouns. 
mass nouns come out of the lexicon with plurality already built in, they are not pluralized morpho-syntactically. Mass nouns with substance readings are also assumed to denote sets of atoms. For instance, mud denotes the set of minimal elements of mud closed under sum. A difference between mass collection terms and substance mass nouns is that minimal elements are perceptually salient in the case of mass collections like furniture while they are not salient in the denotations of substances like mud.

Adopting the single domain strategy of Chierchia (1998), Rothstein (2010) proposes that root nouns are all lexically plural and that mass nouns are root nouns, having plural denotations. In her analysis, the interpretations of count nouns are more complex. She makes uses of the notion of 'M-ATOM' (measure atom), an element in the denotation of a root noun counted as one by some explicit criterion of measurement. The denotation of a singular count noun is derived by an operation of picking out M-ATOMS. When the denotation of a count noun is naturally atomic, as is the case with boy, the M-ATOM operation gives a set of individuals. This interpretation is not affected by contexts. However, when a singular noun is not naturally atomic, its interpretation is context-dependent. For instance, two adjacent stretches of fencing may count as one fence or two fences depending on criteria such as spatial continuity or ownership. Hence, the M-ATOM operation is context-dependent in the case of fence, and what kind of elements are included in the denotation of fence varies depending on contexts.

To implement the context-dependent interpretations of count nouns, Rothstein introduces the measure function 'MEAS,' a function from individuals into ordered pairs consisting of a natural number and a unit of measurement U. Then, M-ATOM is defined to be a function of type $<<e, t><e, t>>$ from sets into sets which maps a set onto a subset of entities which count as one by MEAS. In other words, the M-ATOM operation can be considered as a maximalization operation, giving the set of maximal non-overlapping 
elements which count as 1 entity by a specified unit of measure. Given the notion of MEAS, the interpretations of mass and count nouns are formally distinguished as in the following:

(11) Mass noun: $\lambda x[P(x)]$ Count noun: $\lambda x[\mathrm{P}(\mathrm{x}) \wedge \operatorname{MEAS}(\mathrm{x})=<1, \mathrm{U}>]$

The interpretations of naturally atomic count nouns like boy and nonatomic count nouns like fence are distinctively represented as follows:
a. $[[$ boy $]]=\lambda x[\operatorname{BOY}(x) \wedge \operatorname{MEAS}(x)=<1, \mathrm{BOY}>]$
b. $[[$ fence $]]=\lambda x[\operatorname{FENCE}(\mathrm{x}) \wedge \operatorname{MEAS}(\mathrm{x})=<1, \mathrm{U}>]$

When a noun is naturally atomic as in (12a), the value of $U$ is determined by the meaning of the predicate itself, and M-ATOM is the identity function on the set of naturally atomic individuals. When the predicate is not naturally atomic as in (12b), then value of $U$ is contextually determined.

\subsection{A Revised Domain of Events: Rothstein (2008a)}

In much literature on events including Bach (1986) and Krifka (1986, 1992), it is assumed that the telicity of verbal predicates is closely related to the atomicity of their denotations. Telic predicates such as achievements and accomplishments denote sets of atomic events while atelic predicates of states and activities denote sets of nonatomic events. This correlation is possible since the interpretation domain is separated into atoms and nonatoms. However, Rothstein (2008) argues against this correlation based on her single domain with M-ATOMS.

The starting point of Rothstein's analysis is that all verbal predicates are inherently count, denoting sets of M-ATOMS. ${ }^{4}$ 
(13) Verbal predicate: $\lambda \mathrm{e}[\mathrm{P}(\mathrm{e}) \wedge \operatorname{MEAS}(\mathrm{e})=<1, \mathrm{U}>]$

According to Rothstein (2010), only mass expressions have root meanings and the denotations of count expressions are derived from the application of MEAS. While a value for $U$ in MEAS is lexically determined in the case of naturally atomic nouns, it contextually determined in other cases. Rothstein (2008) argues that a value for $U$ is lexically provided only in the case of telic predicates. Furthermore, she argues that the telicity of verbal predicates are compositionally derived in English and that VPs should be the basic units in determining telicity. An exception to this is achievements. They are naturally atomic, and thus their telicity is determined by the lexical meanings of verbal heads.

Contrasted with telic predicates, the denotations of atelic predicates are derived by an additional function of 'S-summing.' The lattice-theoretic domain suggested by Link (1983) includes a summing operation, which maps two individuals (atoms or sums) to their sum. Rothstein (2008) proposes a separate summing operation of S-summing, which yields a singular entity with the sum of two entities. S-summing defined in the verbal domain is represented as follows:

(14) $\mathrm{S}_{\text {-sum }}$ (= the $\mathrm{S}$-sum operation in the verbal domain): $\forall \mathrm{e} \forall \mathrm{e}^{\prime}\left[\mathrm{P}(\mathrm{e}) \wedge \mathrm{P}\left(\mathrm{e}^{\prime}\right) \wedge \mathrm{R}\left(\mathrm{e}, \mathrm{e}^{\prime}\right) \wedge\left[\mathrm{S}-\operatorname{sum}\left(\mathrm{e}, \mathrm{e}^{\prime}\right) \rightarrow \mathrm{P}\left({ }^{\mathrm{S}}\left(\mathrm{e}+\mathrm{e}^{\prime}\right)\right)\right]\right]$

"For any two events e and e' in the denotation $\mathrm{P}$ which stand in the $\mathrm{R}$ relation, $\mathrm{S}-\mathrm{sum}_{\mathrm{V}}$ applied to e and $\mathrm{e}^{\prime}$ yields a singular event formed out of the sum of e and $e^{\prime}$ and which is also in the denotation of P."

4 Rothstein (1999) argues that the mass/count distinction in the domain of events is observed by the distinction of adjectives and verbs. Adjectives (or APs) have mass denotations and verbs (or VPs) have count readings. She provides several tests to show this difference. 
S-sumv applies to two events in the denotation of a verbal predicate $\mathrm{P}$ which stand in the appropriate relation, sums them, and turns them into a single event also in the denotation of the predicate P. The appropriate relation here is usually understood as temporal overlapping of events and sharing of participants. ${ }^{5}$ For instance, the event of John's running from 9 am till 10 am and that of his running from 10 am till 11 am are temporally overlapped and thus they can be S-summed into a single event of John's running from 9 am till 11 am.

Rothstein assumes that atelic predicates of activities denote sets of minimal events that are defined by intervals and argues that they may be S-summed to form a new singular event as exemplified above. As far as events are stretchable or extended indefinitely, S-summing may apply to them. This means that the events of states may also be S-summed to make a singular event. For instance, two events of John's loving Mary which are temporally adjacent may be S-summed into a single event of John's loving Mary. However, Rothstein claims that there is no distinction between state predicates denoting the set closed under S-summing and state predicates denoting sets of minimal state events because minimal state events are not naturally atomic. Rothstein postulates dense time for states. Hence, even if minimal states hold at instants, they may be split into two smaller states because instants may be further split due to the nature of density. ${ }^{6}$

Finally, achievements may be subcategorized into two categories

5 The condition of temporal overlapping blocks the application of S-summing to achievements and accomplishments. These telic predicates involve a change of events and thus they cannot stand in a temporal overlapping relation. For example, John cannot arrive at the same place twice unless he leaves after the first event and before the second event. Similarly, the same house cannot be constructed twice unless it is taken to pieces after the first event and before the second event begins. So events of change cannot normally be S-summed.

6 Rothstein (2008) does not provide an explanatory account for why the lack of natural atomicity should play a crucial role in the application of S-summing. 
depending on the possibility of repeatedness. Predicates involving a change of states such as arrive cannot be repeated in a given time. When they are repeated, they are understood as separate events. On the other hand, achievements of instantaneous activities such as jump and kick may be repeated and followed by a durational adverbial like for 10 minutes. The latter is called 'semelfactives.' Although Vendler (1967) does not distinguish semelfactives from achievements, they are treated separately depending on theories. Rothstein puts more focus on the repeatedness of semelfactives rather than their instantaneity of individual actions. Hence, she argues that semelfactives are a subcategory of activities. When a semelfactive predicate denotes a set of minimal activity events, it has a semelfactive reading. On the other hand, when it denotes a set closed under S-summing, it has an activity reading.

\subsection{Problems with Rothstein (2008)}

Rothstein (2008) provides an interesting analysis on the semantics of predicates, based on her revised interpretation domain. Although her analysis appears to be useful in accounting for related issues such as the 'imperfective paradox' and the semantics of the progressive aspect, it has a few problems.

First, the role of natural atomicity is not consistent in her theory. In her other paper, Rothstein (2010), natural atomicity is clearly a separate notion from semantic atomicity. The denotations of mass nouns like furniture may be constituted of discrete objects, and those of count nouns like bouquet may be affected by contexts. However, Rothstein (2008) claims that the semantics of verbal predicates hinges on their natural atomicity. Since achievements are naturally atomic, lexical meanings of their verbal heads determine a value for $U$ in MEAS. In other cases, a value for $\mathrm{U}$ is compositionally derived on the level of VP. Moreover, she claims that the application of S-summing is vacuous for states 
because they are not naturally atomic. It is not explained why natural atomicity should be visible only in the event domain. If both nominal and verbal predicates are construed in a domain with M-ATOMS, it needs solid justification to use the same notion of natural atomicity in different ways.

Second, the minimality of nonatoms has been one of the problems with an interpretation domain. Although the notion of M-ATOM appears to contribute to resolving the minimality problem, the ontology of events in Rothstein (2008) still has a problem with minimal entities. She postulates dense time for the semantics of states and argues that states are not naturally atomic because instants of state events may be further split. If she assumes a dense structure for states, she needs a further mechanism to get around the minimality problem. Minimal states cannot be defined in the dense structure.

Third, the operation of S-summing plays a crucial role in the semantics of activities. All verbal predicates are count expressions and their atomicity is defined by MEAS. Since a value of $U$ in MEAS is compositionally derived by the description of a VP, any event of activity predicates needs to be atomic in principle. This is why Rothstein proposes a new operation of S-summing in addition to the ordinary summing operation. S-summing, however, makes a lattice-theoretic domain quite complex and does not capture relations between events properly. Because both summing and S-summing are allowed in a domain, there are two sets of events, one derived by summing and the other derived by S-summing. Suppose that there are two events of John's running from 9 am till 10 am and his running from 10 am till 11 am. Then, these two events may be S-summed to make a singular event of John's running or summed to make a plural event. The former is needed for a sentence like (15a) while the latter is required for the interpretation of (15b). 
(15) a. John ran from 9 am till 11 am.

b. John ran for an hour twice.

This means that all the events of activities are represented by a pair of singular and plural events, which makes the interpretation domain quite complex. A more serious problem is that relations between events are not properly captured. When two events $e_{1}$ and $\mathrm{e}_{2}$ are $\mathrm{S}$-summed to a singular event $\mathrm{e}_{3}$, these three events are all atoms. The part-of relation which should hold between $\mathrm{e}_{1}$ and $\mathrm{e}_{3}$ is not specified in this structure. Likewise, no relation holds between $\mathrm{e}_{1}+\mathrm{e}_{2}$, the sum of $\mathrm{e}_{1}$ and $\mathrm{e}_{2}$, and the S-summed event $\mathrm{e}_{3}$.

To constrain the absurd application of S-summing, Rothstein makes the temporal overlapping condition part of the definition of S-summing. Although a condition like temporal overlapping may be inevitable for S-summing, it needs more consideration.

(16) John ran 300 kilometers this year.

The event described by (16) should be a single M-ATOM according Rothstein's analysis. However, the event of John's running 300 kilometers cannot be generated from temporally overlapped events in a strict sense. Rather, the S-summed event for (16) will be generated from events that are temporally scattered over the year. This shows that a more flexible notion of $\mathrm{R}$ is required for S-summing. Then, the problem is how to control the flexibility of R. R needs to be quite strict in some cases, and it needs to be flexible in others.

Finally, Rothstein's analysis does not provide a mechanism to account for different acceptability of events.

(17) a. John ran/??walked twice.

b. John ate/??slept twice. 
All the verbal predicates in (17) are activities. This means that there should be no differences in the acceptability of the sentences according to Rothstein (2008). However, the occurrences of ran and ate sound much more natural than those of walked and slept. To account for the different acceptability found in (17), not only lexical categories but also contexts need to be considered in determining event types.

\section{A Domain with Context-Dependent Events}

\subsection{A Domain with Context-Dependent Individuals: Chierchia (2010)}

As pointed out in section 3.1, Link's (1983) double domain is not equipped with proper mechanisms to deal with the minimality problem of mass noun denotations, which may vary by contexts. To resolve this problem, Chierchia (2010) resorts to the notion of 'vagueness,' which is extensively discussed by Kennedy (2007) for the semantics of gradable adjectives like tall. Tallness is not absolutely determined but pinned down to a set of degrees of height which are larger than a standard degree for the height in a given context. Different standards depending on contexts provide an explanatory ground for different heights required for tall adults and tall children. The notion of vagueness is needed in the semantics of gradable adjectives because there could be some degrees which are hard to judge for their values even if a standard degree is provided by a context. ${ }^{7}$ For instance, there could degrees

7 Vagueness is formally implemented with the notion of a truth value gap. As for a property $\mathrm{P}$ in a context $\mathrm{c}$, there is a set of entities in the positive extension of $\mathrm{P}$ as well as another set in its negative extension in the context $\mathrm{c}$. In addition to positive and negative extensions, there could be a set of entities that do not belong to either of the sets in this context. 
of height which are difficult to say whether they are tall or short.

Based on the vague semantics of gradable adjectives, Chierchia (2010) argues that while count nouns have a natural cut-off point for their properties, mass nouns may not. 8 We do not hesitate to determine the natural cut-off point for apple or the minimal size of apples. However, there is no unanimous cut-off point or minimal size for the denotation of mass nouns like water or rice. Moreover, the minimal size for rice may vary depending on contexts as discussed in section 3.1. In other words, while atoms for count denotations are stable through contexts, entities for mass denotations are unstable and affected by contexts. Hence, Chierchia argues that stable atomicity is the feature dividing count and mass nouns.

According to Chierchia, mass nouns denote sets of unstable atoms, which are vague entities affected by contexts. Since they are vague, unstable atoms of mass nouns may have a truth value gap when contexts are not precisified enough to clear their

\section{P's Vagueness Band}

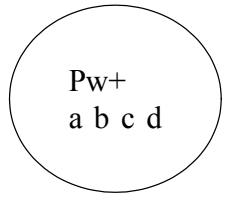

positive extension

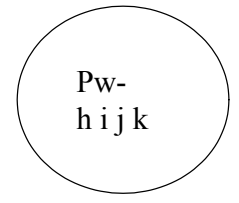

negative extension

When the context $\mathrm{c}$ is more precisified or sharpened successively, Pw may reach a point to be a total function by which no entity remains in the gap.

8 Chierchia (2010) argues that even count noun denotations may not be free from vagueness. For instance, in the situation of cat breeding that performs genetic engineering in search of new breeds, there should be some point that determines the cathood of genetically altered descendants. New breeds of animals that have cat properties more than the requirements of this cut-off point are judged to be cats; the others will be considered as non-cats. However, once the atomicity of cat is determined by the cut-off point, it is not affected by contexts. Chierchia calls this kind of vagueness 'inherent vagueness.' 
vagueness. Then, an interpretation based on a vagueness model is defined as follows:

(18) A vagueness model $\mathrm{M}$ is a tuple of the form $<\mathrm{U}, \mathrm{W}, \mathrm{C}, \propto$, $\mathrm{F}>$, where $\mathrm{U}$ is the set of individuals, $\mathrm{W}$ the set of worlds, $\mathrm{C}$ the set of contexts, $\propto$ a partial order over $\mathrm{C}$, and $\mathrm{F}$ the interpretation function.

Contexts that are minimal with respect to $\propto$ are called 'ground' contexts, and contexts that are maximal relative to $\propto$ are called total precisifications. In this model, a property $\mathrm{P}$ is defined relative to a context. The set of entities in the positive extension of $\mathrm{P}$ may vary by the order of a context.

Given the notion of vagueness and context-dependent atomicity, atoms are now divided into stable and unstable ones. Unstable atoms but not stable atoms are affected by contexts. A vague domain of Chierchia is exemplified as in (19).

(19) The structure of the domain $U$ (relative to a context c)

\begin{tabular}{|c|c|}
\hline \multicolumn{2}{|c|}{ STABLE ENTITIES } \\
\hline $\begin{array}{c}\text { STABLE SUMS } \\
\mathrm{a}+\mathrm{b}+\mathrm{c}\end{array}$ & $\begin{array}{c}\text { PARTIAL SUMS } \\
\mathrm{p}+\mathrm{q}+\mathrm{r}+\mathrm{s}\end{array}$ \\
\hline $\begin{array}{c}\cdots \\
a+b a+c b+c\end{array}$ & $\begin{array}{c}\cdots \\
p+q \\
r+s\end{array}$ \\
\hline $\begin{array}{l}a \mathrm{~b} c \\
\text { STABLE ATOMS }\end{array}$ & $\begin{array}{l}\text { UNSTABLE ENTITIES } \\
\begin{array}{llll}\mathrm{p} & \mathrm{q} & \mathrm{r} & \mathrm{s}\end{array}\end{array}$ \\
\hline
\end{tabular}

In a context c, stable and unstable atoms making the lowest level of a lattice are determined. Stable and partial sums are made 
with these atoms for count and mass denotations. Both count and mass nouns have denotations of sums in the same lattice domain, which are only differentiated by the stability of their entities.

To account for the different acceptability of packaging and grinding readings as discussed in section 3.1, Chierchia proposes that the mapping functions of universal grinding and packaging should be context-dependent just as nominal properties are interpreted with respect to a context. Here is the 'standard S-partition function $\left(\Pi_{\mathrm{ST}}\right)$ ' for the packaging effect.

(20) For any model $\mathrm{M}$, any $\mathrm{c} \in \mathrm{C}$ and any $\mathrm{P} \in \mathrm{D}<\mathrm{e}, \mathrm{t}>$, $\mathrm{F}\left(\Pi_{\mathrm{ST}}\right)(\mathrm{c})(\mathrm{P})$ is the partition for $\mathrm{P}$ most salient in $\mathrm{c}$ (the standard S-partition).

When the values of a context and a property are determined, the function $\Pi_{\mathrm{ST}}$ gives a set of atoms that have the properties and are packaged by the standardized unit in the given context. For instance, when the context sets 'bottle' for the standardized unit of beer, the packaged NP three beers is construed as three bottles of beer. On the other hand, when 'glass' is the standardized unit selected in the given context, three beers means three glasses of beer. If a context does not provide any value for $\Pi_{\mathrm{ST}}$, three beers is not defined.

The grinding effect is achieved by the 'standard i-partition function $\left(\pi_{\mathrm{ST}}\right)$.' The i-partition function $\pi$ is to divide objects into parts, and the standard i-partition function is defined to be $\pi$ with an additional variable of context.

(21) For any model $\mathrm{M}$, any $\mathrm{c} \in \mathrm{C}$, any $\mathrm{P} \in \mathrm{D}<\mathrm{e}, \mathrm{t}>$, and any $\mathrm{u}$ :

i. $\quad F\left(\pi_{\mathrm{ST}}\right)(\mathrm{c})(\mathrm{P})(\mathrm{u})=1$, if $\mathrm{u} \leq \mathrm{c} \pi(\cup \mathrm{P})=1$ and $\mathrm{u}$ is of size $\mathrm{s}(\mathrm{c})$

ii. $\mathrm{F}\left(\pi_{\mathrm{ST}}\right)(\mathrm{c})(\mathrm{P})(\mathrm{u})=0$, if it is not the case that $\mathrm{u} \leq \mathrm{c} \pi$ $(\cup \mathrm{P})$

iii. $F\left(\pi_{S T}\right)(c)(P)(u)$, undefined otherwise 
where $\pi$ most salient i-partition in $\mathrm{c}$ such that $\pi(\cup \mathrm{P}) \subseteq \mathrm{P} \Sigma$, if there is such a $\pi$.

When the values of a context and a property are determined, $\pi_{\mathrm{ST}}$ assigns a positive value to a set of unstable entities if they have that property and their size is identifiable in the context. When their size is not defined in the context, unstable entities are not assigned any truth value even if they meet the requirement of the property.

The context-dependency of the functions is useful to explain different degrees of acceptability for shifted readings of nouns. According to Chierchia's analysis, how easily packaged or ground readings are allowed depends on how many contexts are available for those readings. For instance, the number of contexts for the packaged reading of beer is understood to be larger than that of blood. Hence, three beers is much easier to get than three bloods by $\Pi_{\mathrm{ST}}$. In the same way, the shifted reading of apple is more widely used than that of table. This means that there are more contexts for the ground entities of apple than those of table. Hence, the shifted reading of apple is more easily allowed by $\pi_{\mathrm{ST}}$.

\subsection{A Proposal for a Domain with Context-Dependent Events}

An interpretation domain provides a basis on which each expression is assigned a proper meaning. This role of an interpretation domain drives us to take a holistic view on a domain. Hence, if we accept Davidson's (1967) theorum that events are individuals just like objects, it is better to assume the same structure for objects and events, following the argument of Bach (1986) and Krifka (1986, 1992). Given the similarity required for the structures of objects and events, the first step to take in determining the semantics of verbal predicates is to compare interpretation domains for nominal predicates and select a proper domain for them. And then, we need to build up a 
similar structure for events in accord with the ontological properties of nominal denotations.

To overcome the problems of the double domains as proposed by Link (1983), two analyses are reviewed in this study: Chierchia (2010) and Rothstein (2010). Although Rothstein's domain is more flexible than Link's, it still does not cope properly with the problems such as the minimality of entities and the contextdependency of expressions, especially with shifted readings. It also lacks theoretical consistency by applying the notions of natural and semantic atomicity distinctively to nominal and verbal predicates. In contrast, Chierchia proposes a vague domain with contextdependent entities of unstable atoms. The context-dependent properties of mass denotations provide an appropriate solution to the minimality problem and contextually affected interpretations. Therefore, I will delve into the interpretations of verbal predicates in the framework of Chierchia.

The semantics of achievement predicates (other than semelfactives) is quite simple because they concern themselves with the change of states. According to the contextual dependency of Chierchia's domain, how short time is relevant in defining states may vary depending contexts. However, once a contextual variable is set, relevant time spans for states will be determined. Then, the semantic role of achievements is only to mark a change between states. Hence, it is natural to assume that achievements denote sets of stable atoms describing state changes.

The stability of achievements is supported by plurality of their events. Note that stability is the main feature of count expressions in Chierchia's domain, and that the availability of plural forms is one of the morpho-syntactic properties of count expressions. Then, to argue for the stability of achievements. we need to show whether plurality is allowed to them. Since English does not include explicit markers for plural predicates, we may resort to the plural interpretations of achievements. When a plural subject accompanies an achievement, the sentence interpretation may 
result in a plural event.

(22) They arrived separately, one at $1 \mathrm{pm}$ and the other at $2 \mathrm{pm}$.

When two people arrive at some place, their arrival may be done simultaneously or separately. The separate arrival of people may have different temporal values as in (22). This shows that the event in (22) is a sum of two events and that the predicate is a count expression.

Accomplishment predicates are durative, describing events which take time. As telic predicates, accomplishments have lexically designated goals, which are achieved by various subevents. For instance, the event of building a house is completed only when a new house comes to exist as a result of various activities such as setting up walls, constructing a roof, and so on. The relation of an event and subevents is the replicate of a relation between a group like committee and its members. In view of the fact that group-member relations are captured by a group formation function according to Link (1984) and Landman (1989), we assume that the same semantic operation is also applicable to events. Thus, we assume that accomplishments denote sets of group events which are generated from multiple subevents via the group formation function. Since groups are postulated as atoms in Link and Landman, we also assume that group events are stable in the framework of Chierchia.

The events of states and activities are extendable and cumulative. The event of a state loving for a year may be extended to the state of two years' loving, or the events of loving this year and last year may be cumulatively denoted by loving for two years. Likewise, the event of an activity like run also shares semantic properties with a state in its extendedness and cumulativity. Since states and activities are easily extended and cumulated, it is hard to pin down minimal events for them. Minimality is at issue here 
just like mass nouns. Hence, it is plausible to postulate that states and activities denote sets of unstable events. Since the atomicity of unstable entities are contextually determined in Chierchia's domain, the size of events or the temporal value of events like John's loving Mary or John's running is vague and determined by contexts.

One of properties of unstable entities is that they may be packaged to stable entities by the standard S-partition function. The contextual dependency of $\Pi_{\mathrm{ST}}$ accounts for the different acceptability in (23a).

(23) a. three beers/??bloods

b. John ran/??walked twice.

c. John ate/??slept twice.

The occurrence of the numeral adverbial twice in (23b) and (23c) shows that the predicates in these sentences are count. To be count, the unstable events of the activities should be packaged to stable events by $\Pi_{\text {ST. The stability of running events is more }}$ acceptable than that of walking events because there will be more contexts to package running events, say athletic games. Similarly, the packaged construal of eating is acceptable in the sense of eating a meal. However, it is not easy to come up with contexts to package sleeping events. The different acceptability of activities is well explained in Chierchia's domain.

Finally, semelfactives like jump and kick are categorized as achievements and assumed to denote sets of stable events. Vendler (1967) focuses on the instantaneity of semelfactive events and classifies them as achievements. However, Rothstein (2008) puts more emphasis on their durative property in repeated readings and thus classifies them as activities. I argue that semelfactives are count expressions unlike activities. 
(24) a. John jumped for 10 minutes. He jumped 100 times in total.

b. John ran for 10 minutes. \#He ran 100 times in total.

Although both jumping and running may be extended and take time as specified by the durative adverbial for 10 minutes, they have different event structures. The event of jumping for 10 minutes may be paraphrased by the event with a numeral expression as in (24a), the event of running is not allowed to be paraphrased in this way. Hence, it is more persuasive to assume that semelfactives are achievements and denote sets of stable events.

\section{Conclusions}

The introduction of new entities of events by Davidson (1967) has the effect of expanding the horizon of semantic research and casts an interesting question of how far the interpretations of nominal and verbal predicates should show similarities. Based on Davidson's theorum that events are individuals just like objects, it is widely accepted that the event domain should be generated from the same structure as that of the object domain as argued by Bach (1986) and Krifka (1986, 1992).

Since the seminal work of Link (1983), in which objects are structured into a join semi-lattice with atoms and sums, several ontological problems are raised in semantics: the minimality of nonatoms, relations between natural and semantic atomicity, and contextually affected shifted readings. To deal with these problems, Chierchia (1998) proposes a single domain with atoms only and Rothstein (2010) elaborates this domain with the notion of measured atoms (M-ATOMS). Based on the revised domain of objects, Rothstein (2008) puts forward an analysis on the 
semantics of verbal predicates. She argues that verbal predicates are all count expressions to denote M-ATOMS and proposes a new operation of S-summing for the semantics of activities. She also emphasizes the extendedness of semelfactive predicate and classifies them as activities.

Although Rothstein's analysis appears to be interesting in some aspects related with the aspectual properties of verbal categories, it is based on an interpretation domain which does not cope with the minimality problem and the different acceptability of shifted readings. Hence, her arguments on the semantics of verbal predicates are not exempt from the same problems.

As an alternative to this analysis, I adopt a vague interpretation domain with stable and unstable entities as proposed by Chierchia (2010) and argue that only achievements and accomplishments are count expressions. Achievements denote sets of stable events while accomplishments denote sets of group events, which are also stable. States and activities show semantic properties similar to those of mass nouns, namely extendedness and cumulativity, and thus they are assumed to denote sets of unstable events. Just like unstable atoms of mass nouns, minimal events for states and activities are contextually determined. Finally, I focus on the countability of semelfactives and argue that they are achievements.

\section{References}

Bach, E. 1986. The Algebra of Events. Linguistics and Philosophy 9, 5-16.

Chierchia, G. 1998. Plurality of Mass Nouns and the Notion of

Semantic Parameter. In S. Rothstein (ed.), Events and Grammar. Dordrecht: Kluwer. . 2010. Mass Nouns, Vagueness, and Semantic Variation. Synthese 174, 99-149. 
Davidson, D. 1967. The Logical Form of Action Sentences. In N. Resher (ed.), The Logic of Decision and Action 81-95. Pittsburgh: University of Pittsburgh Press.

Kennedy, C. 2007. Vagueness and Grammar: The Semantics of Relative and Absolute Gradable Adjectives. Linguistics and Philosophy 30, 1-45.

Krifka, M. 1986. Nominal Referenz und Zeitkonstitution: Zur Semantik von Massentermen, Individualtermen, Aspektklassen.

Ph.D Dissertation. The University of Munich. 1992. Thematic Relations as Links between Nominal Reference and Temporal Constitution. In I. Sag \& A. Szabolsci (eds.), Lexical Matters 29-53. Stanford: CSLI.

Landman, F. 1989. Groups I \& II. Linguistics and Philosophy 12, 559-606 \& 723-744. - 1996. Plurality. In S. Lappin (ed.), A Handbook of

Contemporary Semantic Theory 425-457. Oxford: Blackwell. Lasersohn, P. 1995. Plurality, Conjunction, and Events. Dordrecht: Kluwer Academic Publishers.

Link, G. 1983. The Logical Analysis of Plurals and Mass Terms: A Lattice Theoretical Approach. In N. Bäuerle et al. (eds.), Meaning, Use, and Interpretation of Language 302-323. Berlin: Walter de Gruyter.

. 1984. Hydras and the Logic of Relative Construction with Multiple Heads. In F. Landman et al. (eds.), Varieties of Formal Semantics 245-257. Dordrecht: Foris.

Parsons, T. 1990. Events in the Semantics of English. Cambridge, MA: MIT Press.

Rothstein, S. 1999. Fine-Grained Structure in the Eventuality Domain: The Semantics of Predicate Adjective Phrases and Be. Natural Language Semantics 7, 37-420. 2008. Telicity, Atomicity, and the Vendler Classification of Verbs. In S. Rothstein (ed.), Theoretical and Crosslinguistic Approaches to the Semantics of Aspect 43-78. Amsterdam: John Benjamin. 
84 An Interpretation Domain with Context-dependent Events

. 2010. Counting and the Mass/Count Distinction. Journal of Semantics 27, 343-397.

Schein, B. 1995. Plurals and Events. Cambridge, MA: MIT Press.

Vendler, Z. 1967. Linguistics in Philosophy. Ithaca: Cornell. 